\title{
OTIONOMICS
}

Revista de economía, empresa y sociedad

Dossier: «Repensando la enseñanza de la economía en la universidad»

\section{Rebelión en las aulas de economía: por qué poner fin a la monocultura neoclásica}

\section{Ferran Fspañol Gasanovas}

Graduado en Economía y estudiante de Física

\section{Laura de la Villa Aleman}

Investigadora predoctoral del Departamento de Historia e Instituciones Económicas (Universidad de Barcelona)

RESUMEN La economía es la única disciplina académica que cada día tiene más estudiantes en rebeldía por estar en contra de los contenidos que se ofrecen en las universidades. Lo que pretendemos con este artículo es mostrar el alcance del problema que hay detrás de este descontento, abordando con más detenimiento algunas de sus dimensiones. En primer lugar, se define el estado actual de la disciplina económica como una monocultura basada en los tres principios axiomáticos de la economía neoclásica y se discute el problema de la monocultura desde el debate epistemológico de fondo. En segundo lugar, se muestra la falta de pluralismo en los actuales planes de estudio mediante los resultados de los análisis hechos por distintos colectivos para los casos de las universidades del Reino Unido, Francia y España. En tercer lugar, se presenta el pluralismo de teorías, de disciplinas y de metodologías como estrategia para romper con la monocultura neoclásica y mejorar la formación de los estudiantes de economía. El artículo concluye poniendo de relieve que el pluralismo en la enseñanza de la economía es una necesidad no solo para los estudiantes, sino también para la academia, el mundo de los negocios y la sociedad en general.

PALABRAS CLAVE educación económica; teoría económica; metodología ciencias sociales; interdisciplinariedad; pluralismo

\section{Rebellion in economics classrooms: why the neoclassical monoculture is to be ended}

SUMMARY Economics as an academic discipline is unique inasmuch as ever-increasing numbers of students are rebelling against the content offered by universities. Our intention with this paper is to expose the scope of the problem lying behind this discontent, addressing some of its aspects in close detail. First, the economics discipline is defined in its current state as a monoculture based on the three axiomatic principles of neoclassical economics, and through epistemic discourse, the monoculture problem is discussed in depth. Second, the lack of pluralism in current study programmes is highlighted, with the results from analyses conducted by a variety of groups focusing on universities in the United Kingdom, France and Spain. Third, the pluralism of theories, disciplines and methodologies is presented as a strategy to break with the neoclas- 
sical monoculture and to improve the education of students of economics. The paper concludes by bringing into sharp focus how pluralism in the teaching of economics is necessary not only for economics students, but for the academic world, the business world and society in general.

KEYWORDS economics education; economic theory; social sciences methodology; interdisciplinarity; pluralism

\section{Introducción}

La economía es la única disciplina académica que cada día tiene más estudiantes en rebeldía por estar en contra de los contenidos que se ofrecen en las universidades. La primera carta abierta que reclamaba un cambio en los planes de estudio se publicó en junio del año 2000 de la mano del movimiento de estudiantes francés PostAutistic Economics ${ }^{1}$. En su declaración pedían una salida de los mundos imaginarios que les enseñaban en las aulas para poder entender cómo funcionan las economías reales.

En noviembre de 2011, un grupo de estudiantes de la Universidad de Harvard se retiró en señal de protesta de una lección magistral de Gregory Mankiw², autor de uno de los manuales de principios de economía más utilizados en los grados de economía de todo el mundo. Denunciaban que generaba un sesgo en la formación introductoria a la economía, que faltaba una revisión crítica de los conocimientos que se transmitían y que todo ello conllevaba graves consecuencias tanto para los estudiantes como para el conjunto de la sociedad.

La noticia dio la vuelta al mundo y ese mismo año se producía una conferencia organizada por el Banco de Inglaterra con el título Are Economics Graduates Fit for Purpose?, que resultó ser la chispa para la posterior creación de Post-Crash Economics Society en Manchester a lo largo del curso 2012-2013³. Los estudiantes de Manchester denunciaron la falta de herramientas para entender la crisis económica que había estallado a finales del 2007 y los problemas económicos en general, y pusieron en marcha campañas y proyectos de formación al margen de los cursos de la universidad. Pronto empezaron a aparecer nuevos grupos en varias universidades británicas y resultó ser el inicio de ISIPE.

El 5 de abril de 2014 se presentó al mundo el llamamiento fundacional de ISIPE (International Student Initiative for Pluralism in Economics) ${ }^{4}$. Bajo estas siglas, cuarenta y dos asociaciones de estudiantes de más de diecinueve países pusieron de manifiesto la necesidad de entender que no solamente la economía estaba en crisis, también los planes de estudio de economía lo estaban. La falta de pluralidad intelectual, las limitaciones de las herramientas analíticas enseñadas y la necesidad de devolver el mundo real a las aulas fueron las ideas que lograron captar la atención de los principales medios de comunicación de todo el mundo. Casi dos años después del llamamiento, seguimos aumentando el número de colectivos, académicos, profesionales e instituciones que se suman a la reivindicación.

Lo que pretendemos con este artículo es mostrar el alcance del problema que hay detrás de este descontento, abordando con más detenimiento algunas dimensiones. En la segunda sección caracterizamos el estado actual de la disciplina económica como una monocultura basada en los principios axiomáticos de la economía neoclásica. En la sección 3 mostramos la falta de pluralismo en los actuales planes de estudio mediante los resultados de los análisis existentes para los casos de las universidades del Reino Unido, Francia y España. En la

1. "Open letter from economic students to professors and others responsible for the teaching of this discipline», 2000. Disponible en línea: <http://www.paecon.net/PAEtexts/a-e-petition.htm>.

2. "Carta abierta a Gregory Mankiw», 2011. Disponible en línea: <http://www.economiacritica.net/?p=732>.

3. Véase: <http://www.post-crasheconomics.com/about_us/>.

4. «Llamamiento internacional de estudiantes de Económicas a favor de una enseñanza pluralista», 2014. Disponible en línea: <http:// www.isipe.net/home-es>. 
sección 4 desarrollamos el contenido de la reivindicación por el pluralismo y algunas de las barreras a superar. Y en la sección 5 , se concluye.

\section{La monocultura de los axiomas neoclásicos}

La reivindicación del cambio en los planes de estudio de los grados de Economía parte de una cuestión que concierne al conjunto de la disciplina y no únicamente a su vertiente docente. El problema radica en la centralidad del programa de microfundamentos neoclásicos de la economía. Una centralidad que podría caracterizarse como monopolio intelectual y que relega otras aproximaciones a la marginalidad.

Sin embargo, a día de hoy la escuela neoclásica suele aparecer como una tradición poco definida. De hecho, uno de los argumentos más recurrentes en contra de la reivindicación de la pluralidad en los planes de estudio es la propia negación del neoclasicismo como corriente, dándose la paradoja de que tiene muy pocos adeptos explícitos. Esto nos lleva a la necesidad de conceptualizar qué entendemos por economía neoclásica y lo hacemos distinguiendo los tres principios axiomáticos comunes de este paradigma5: el individualismo metodológico, el instrumentalismo metodológico y el equilibrio metodológico. A partir de estos tres axiomas, todo fenómeno social analizado por la economía neoclásica se explica como un equilibrio resultado de la interacción de individuos con racionalidad instrumental que optimizan la satisfacción de sus preferencias. Esto es así en las teorías de equilibrio general, pero también, entre otras, en la economía industrial, las teorías de elección social, la geografía económica y la nueva economía política.

Estos tres principios metodológicos conforman el núcleo de lo que se enseña durante los estudios de grado y representan las pautas del juego que cualquier economista debe seguir para desarrollar una carrera académica exitosa. Sin embargo, no son discutidos ni en las clases ni en los debates académicos.

Dicho esto, la cuestión principal pasa a ser en qué medida es necesario discutir la base axiomática del análisis económico. Milton Friedman sintetiza la visión positivista dominante en esta cuestión ${ }^{6}$ y defiende que no eran tan importantes los supuestos que se usaban, sino los resultados que se obtenían con ellos. Sin embargo, esta visión fue rebatida por Alan Musgrave en 1981 a partir de una taxonomía que distinguía entre los axiomas de "dominio", los axiomas «negligibles» y los axiomas «heurísticos» en función de la importancia o no de su realismo.

El alegato de Friedman únicamente es aceptable para los axiomas negligibles, es decir, aquellos referidos a elementos que en una primera aproximación son irrelevantes para estudiar un fenómeno. Sin embargo, cuando los axiomas sí consideran cuestiones relevantes para estudiar un fenómeno, su irrealismo limita el poder explicativo de aquella teoría. Y la cuestión es que, de hecho, el instrumentalismo de Friedman subordina el poder explicativo de una teoría a su poder de predicción. El problema es, por un lado, no tener en cuenta que se puede dar una teoría con poder explicativo y sin poder predictivo (como es el caso de los sistemas complejos y la teoría del caos) y, por otro lado, que la verificación de una teoría mediante su capacidad predictiva es sencillamente insuficiente si no se considera en un contexto en el que distintas teorías compiten entre ellas?

Las inconsistencias de la teoría neoclásica que se derivan de estos principios axiomáticos es otro de los aspectos cruciales sistemáticamente omitidos en las lecciones de economía. Uno de los trabajos más útiles a día de hoy para entender los problemas básicos implícitos en los fundamentos de la teoría neoclásica es el libro de

5. Arnsperger y Varoufakis (2006). "What Is Neoclassical Economics? The three axioms responsible for its theoretical oeuvre, practical irrelevance and, thus, discursive power». Panoeconomicus. N. ${ }^{1}$, págs. 5-18.

6. Friedman (1966). «The Methodology of Positive Economics». En: Essays In Positive Economics. Chicago: University of Chicago Press, pág. 14.

7. Popa (2012). «Methodological Assumptions in Economics: The Argument against Instrumentalism». Cogito: Multidisciplinary Res. J. N. ${ }^{\circ}$, pág. 102. 
Steve Keen La economía desenmascaradå. Un ejemplo muy ilustrativo es el del problema de agregación de las demandas individuales: lo que los economistas neoclásicos demostraron como válido para un mundo con un solo individuo deja de serlo para una sociedad. El punto crítico es que en una población de más de un individuo la distribución del ingreso pasa a tener un papel fundamental en la determinación de la demanda de una economía y, por tanto, del ciclo económico.

Entonces, no únicamente es importante adoptar una visión crítica respecto a la teoría económica en tanto que esta derive resultados coherentes con el mundo real, sino que debe construirse en base a principios metodológicos consistentes. Incluso cabe cuestionarse si la axiomatización de la teoría económica es la única vía posible para su desarrollo?.

Parece razonable pensar que la falta de discusión y, por tanto, comprensión de las bases de la economía neoclásica limita la capacidad que tienen los economistas para desarrollar teorías y planteamientos que permitan avanzar a la disciplina. Sin embargo, desde la segunda mitad del siglo xx se ha impuesto una visión acumulativa de la historia del pensamiento económico ${ }^{10}$, una versión del positivismo lógico. Esta aproximación entiende la economía como una ciencia delimitada por el conjunto de proposiciones verdaderas sobre cuestiones económicas y, por tanto, el nuevo conocimiento se agrega a los ya disponibles o los sustituye. De este modo, el estado actual de la teoría económica sería el resultado de un progreso continuo de la disciplina. Consiguientemente, dado que se asume que «la teoría actual es la mejor que nunca ha existido, no hay necesidad de contrastarla con la de sus predecesores»"11. Esta concepción triunfalista del establishment de la economía no solo ha llevado a justificar la falta de competencia entre distintas aproximaciones teóricas y metodológicas, sino también la ausencia de la historia del pensamiento económico en la formación de los economistas.

Como explica Roncaglia ${ }^{12}$, la influencia de algunos historiadores y filósofos de la ciencia, tales como Kuhn, Lakatos y Feyerabend, contribuyó al cuestionamiento de la metodología positivista en las ciencias sociales, dando lugar a la visión competitiva de la evolución del pensamiento económico. Básicamente, lo que rechaza expresamente esta aproximación es la idea de un proceso unidimensional de avance científico, ya que puede haber progreso en el seno de cada enfoque y a lo largo de la sucesión histórica de paradigmas. De hecho, entre los sucesivos paradigmas existen diferencias en la cosmovisión subyacente: a algunos aspectos de la realidad se les da una mayor importancia, y a otros menos, de manera que existen diferencias en el conjunto de supuestos sobre los que están construidas las teorías, y, por tanto, en el ámbito de aplicabilidad de las teorías. Con ello, queda justificada la necesidad de pluralidad y competencia entre enfoques para resolver preguntas económicas.

\section{Los planes de estudio a escrutinio}

El movimiento por el pluralismo en la enseñanza ha dado un paso adelante recientemente en la voluntad de aportar análisis concretos sobre el problema de la formación de los estudiantes de economía. Esto ha llevado a que distintos colectivos comprometidos con esta reivindicación analizaran el contenido de los planes de estudio en distintos informes.

8. Keen (2015). La economía desenmascarada. Madrid: Capitán Swing. Véase: <http://capitanswing.com/libros/la-economia-desenmascarada/>.

9. Nos referimos al programa formalista de David Hilbert, que tenía como objetivo demostrar la consistencia absoluta de las matemáticas, y que fue rechazado por los teoremas de incompletitud del lógico Kurt Godel en 1931.

10. Roncaglia (2006). La riqueza de las ideas: una historia del pensamiento económico (cap. 1, págs. 17-81). Zaragoza: Prensas Universitarias de Zaragoza.

11. Freeman, Chick and Kayatekin (2014). Cambridge Journal of Economics, vol. 38, issue 3, pp. 520-521.

12. Roncaglia (2006). La riqueza de las ideas: una historia del pensamiento económico (cap. 1, págs. 17-81). Zaragoza: Prensas Universitarias de Zaragoza. 
El primer informe fue realizado por la Post-Crash Economics Society de Manchester ${ }^{13}$. Su análisis se centra principalmente en las metodologías de evaluación que se usan en los principales módulos de un grado de economía (especialmente microeconomía y macroeconomía). Este estudio permitió detectar que en dieciocho de los cuarenta y ocho módulos, más del 50\% de la nota dependía de exámenes tipo test (llegando a ser del 100\% en los cursos básicos, donde se enseñan los principios básicos de la economía). A su vez, pusieron de manifiesto que únicamente en once de los cuarenta y ocho módulos aparecían los términos crítica, evaluar o comparar en los planes docentes. Una de las principales conclusiones que remarcan es que «la consecuencia es una educación en economía que forma a los estudiantes para que acepten la teoría económica y la reproduzcan mecánicamente en los exámenes, sin cuestionar los supuestos que hay detrás» (pág. 17). Además, remarcan el poco énfasis en temas de ética, política y filosofía, y que no se les enseña a usar herramientas para valorar si aquello que están aprendiendo tiene o no sentido: es decir, la falta de criterio informado a la hora de escoger una herramienta u otra en función del fenómeno concreto de estudio.

El segundo informe lo hizo la asociación PEPS-Économie en 2014 y analizaba cincuenta y cuatro universidades de Francia ${ }^{14}$. Para realizarlo, los estudiantes franceses categorizaron los títulos de las asignaturas en distintos ejes para medir su peso dentro del conjunto del grado. En el informe muestran que la reflexión epistemológica está prácticamente ausente en los planes de estudio (solo una universidad ofrece una asignatura de este tipo). También destacan que la asignatura de historia del pensamiento económico únicamente se oferta en quince de las cincuenta y cuatro universidades y representa el 1,7\% del total de las clases. Asimismo, las asignaturas de teoría económica solo representan el 0,5\% del total y las de problemas concretos de las economías contemporáneas representan el 1,7\%. Por último, las materias de política y sociología representan tan solo el 1,8\% del total de las clases impartidas.

El último informe en llegar ha sido el de la asociación Post-Crash Barcelona, en el que se analizan todos los grados de economía de las universidades del Reino de España ${ }^{15}$. El informe denota problemas similares a los anteriores: la teoría neoclásica es omnipresente en los planes de estudio, no hay cuestionamiento ni revisión crítica de los axiomas y metodologías, se margina la historia del pensamiento económico y no hay reflexiones interdisciplinares. Además, el informe cuenta con un amplio estudio cuantitativo sobre el contenido de los créditos que componen los cuarenta y cinco grados de economía impartidos en España en el curso 2014-2015. Los resultados obtenidos indican que un $48,14 \%$ de los créditos obligatorios están dedicados a asignaturas relacionadas con los métodos cuantitativos, microeconomía y macroeconomía. Solo se destinan un 4,8\% de los créditos obligatorios a la historia económica, frente al casi 10\% dedicado al ámbito de la empresa. Además, las asignaturas de tipo interdisciplinar o de reflexión epistemológica suponen el 7,3\% del total de créditos obligatorios. Por último, se confirma el elevado grado de homogeneización entre los distintos planes de estudio y se pone de manifiesto su gran similitud con los programas de Administración y Dirección de Empresas.

Las semejanzas en los principales resultados de los tres informes es una prueba más de que la monocultura de los planes de estudio es un fenómeno internacional. La exclusividad de la que goza el paradigma neoclásico en el contenido de los currículos académicos, la gran homogeneidad de la oferta, la falta de perspectiva histórica y la falta de discusión de los principios de la disciplina son, sin duda, los principales titulares.

13. PCES (2014). Economics, Education and Unlearning: Economics Education at the University of Manchester. Véase: <http://www. post-crasheconomics.com/economics-education-and-unlearning/>.

14. PEPS (2014). The case for pluralism: what French undergraduate economics teaching is all about and how it can be improved. Véase: <https://pepseco.files.wordpress.com/2015/05/peps-the-case-for-pluralism-ijpee-2014.pdf>.

15. Post-Crash Barcelona (2015). Informe de la enseñanza de la Economía en las universidades españolas. Véase: <https://postcrash barcelona.wordpress.com/2015/09/03/informe-de-la-ensenanza-de-la-economia-en-las-universidades-espanolas/>. 


\section{Hacia un mayor pluralismo en la docencia de la economía}

El movimiento de estudiantes por un cambio en la enseñanza en la economía reclama una mayor pluralidad en tres aspectos concretos: el pluralismo teórico, el pluralismo metodológico y el pluralismo de disciplinas. Por pluralismo teórico entendemos que la universidad tiene que facilitar la comprensión de las teorías surgidas de las distintas escuelas de pensamiento para conseguir que los estudiantes puedan analizar los fenómenos económicos desde el enfoque más pertinente. Actualmente, las perspectivas institucionalistas, conductuales, marxistas y poskeynesianas están prácticamente ausentes. Cuando reivindicamos un mayor pluralismo metodológico, estamos pidiendo que nos doten de más herramientas que las estrictamente estadísticas y econométricas por más que estas deban tener un peso importante. Necesitamos formación en contabilidad nacional, tenemos que poder analizar las cuentas de flujos de fondos o mejorar nuestra formación en herramientas tan utilizadas como las encuestas y las entrevistas ${ }^{16}$. El tercer pilar es lo que hemos llamado pluralismo interdisciplinario, que persigue el objetivo de abrir un debate y una colaboración estrecha con otras ramas de las ciencias sociales que se solapan con el ámbito de la economía y que nos presentan como entes independientes de los fenómenos que estamos estudiando.

Una de las barreras importantes para la implementación de dichos cambios es el establishment actual dentro de la disciplina. Una modificación de los planes de estudio supone una amenaza a su trayectoria intelectual y profesional. En este sentido, es muy pertinente la metáfora que plantean Ha-Joon Chang y Jonathan Aldred cuando decían ${ }^{17}$ :

«Es como pedirle al clero católico del Medievo que enseñara a sus nuevos estudiantes diferentes interpretaciones de la cristiandad, que dejaran de enseñar exclusivamente en latín para hacerlo en lenguas vernáculas y que animaran a desafiar la autoridad intelectual y moral de la Santa Sede».

Otros argumentos que se usan a menudo para poner barreras al cambio se basan en infantilizar al estudiantado. Ya sea considerando que las críticas a la teoría son demasiado complicadas (cuando algunas de ellas se resuelven en una línea de cálculo, como explica Steve Keen) o que los debates abordados desde diferentes perspectivas pueden llevar a la confusión y la desorientación.

\section{Conclusiones: Romper con la monocultura y avanzar hacia el pluralismo}

La centralidad de la economía neoclásica en los planes de estudio conlleva graves deficiencias en la formación y capacidades de los futuros economistas. Pero este no es un problema exclusivo para aquellos estudiantes que en el día de mañana van a seguir cultivando la ciencia económica desde la academia.

Se están ofreciendo unos títulos universitarios que no cumplen tampoco con las expectativas de los empleadores. Por ejemplo, voces prominentes del Banco de Inglaterra y la City de Londres denuncian la falta de aptitudes de las nuevas generaciones. La formación obtenida es ineficaz para afrontar los retos del mundo del mañana y no tener una noción sólida del funcionamiento de la economía real perjudica enormemente las aspiraciones

16. Ha-Joon Chang y Jonathan Aldred (2014). «Después del crack, necesitamos una revolución en el modo de enseñar la economía». Revista SinPermiso (18/5/2014). Véase: <http://www.sinpermiso.info/textos/la-teora-econmica-y-su-enseanza-en-cuestindossier>

17. Ibidem. 
económicas de cualquier empresa. La falta de pericia comunicativa y la incapacidad de visualizar y resolver problemas reales son algunas de las carencias de los nuevos titulados.

Asimismo, el problema presente en la docencia de la economía se deriva del estado general de la disciplina. Por tanto, el énfasis en el cambio del currículo académico adquiere relevancia para la propia evolución de la disciplina en tanto que el cambio en la formación de nuestros jóvenes tendrá un impacto en los debates académicos del futuro.

Por último, el compromiso con romper la monocultura es esencial, dado que la economía tiene un impacto directo para nuestra cotidianidad. La reivindicación del pluralismo va de la mano de la recuperación de los problemas materiales reales de nuestras sociedades y contribuir a mejorar el debate público.

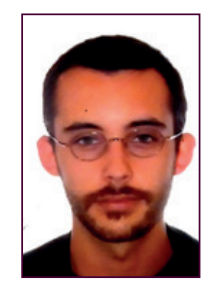

Ferran Fspañol Gasanovas fespanyol@gmail.com Graduado en Economía y estudiante de Física

Graduado en Economía por la Universidad Pompeu Fabra en 2015 y actualmente estudiante del grado en Física por la Universidad de Barcelona. Evaluador y miembro de la Comissió de Ciències Socials i Jurídiques de la Agència de Qualitat Universitària de Cataluña desde 2012. Miembro de la Junta de Facultad de Economía y Empresa del 2010 al 2012. Es uno de los miembros impulsores de la International Student Initiative for Pluralism in Economics en España y del colectivo Post-Crash Barcelona.

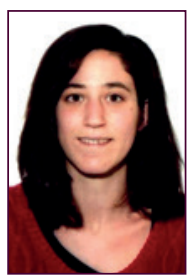

Laura de la Villa Aleman

delavilla.laura@ub.edu

Investigadora predoctoral del Departamento

de Historia e Instituciones Económicas

(Universidad de Barcelona)

Licenciada en Economía por la Universidad Pompeu Fabra en 2012 y máster en Historia Económica por la Universidad de Barcelona, la Universidad Autónoma de Barcelona y la Universidad de Zaragoza en 2013. Desde el 2014, es investigadora predoctoral en el Departamento de Historia e Instituciones Económicas de la Universidad de Barcelona. Dedica su investigación a temas relacionados con la inestabilidad financiera e imparte docencia en la asignatura de Historia Económica Mundial de los grados de Economía y ADE. Implicada desde el inicio de sus estudios con la reivindicación del pluralismo en los planes docentes de economía, ha sido una de las impulsoras de la International Student Initiative for Pluralism in Economics en España y miembro fundador del colectivo Post-Crash Barcelona.

Los textos publicados en esta revista están -si no se indica lo contrario- bajo una licencia Reconocimiento-Sin obras derivadas 3.0 España de Creative Commons. Puede copiarlos, distribuirlos y comunicarlos públicamente siempre que cite su autor y la revista y la institución que los publica (autoría, nombre de la revista, institución editora); no haga con ellos obras derivadas. La licencia completa se puede consultar en http://creativecommons.org/licenses/by-nd/3.0/es/deed.es.

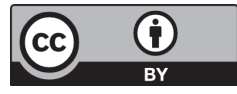

